\title{
The display and animation of full-color images in real time on the Macintosh computer
}

\author{
JOHN A. BARO \\ University of Missouri, St. Louis, Missouri \\ and \\ HOWARD C. HUGHES \\ Dartmouth College, Hanover, New Hampshire
}

\begin{abstract}
Techniques for displaying and animating full-color and gray-scale images in real time are illustrated with Pascal examples. The Palette Manager procedures included in the Macintosh Toolbox are used to circumvent the speed limitations inherent in drawing and redrawing images on the Macintosh's video display. With the technique of palette animation, images of arbitrary size and complexity can be displayed easily and animated at the scan rate of the video monitor. The routines described here are based on Toolbox procedures available on any Macintosh with color video capabilities.
\end{abstract}

The Macintosh has become the computer of choice for a wide variety of graphics-intensive applications. With the availability of color, and very recently, of 32-bit "true" color, the Macintosh now provides a sophisticated graphics environment that is comparable to that provided by many graphics work stations costing several times more. Yet despite these capabilities, and despite the increasingly widespread adoption of the Macintosh for laboratory data acquisition and analysis, its real-time color graphics capabilities remain largely unused by the research community.

One reason for this underutilization is that, unlike most other graphics workstations, the Macintosh lacks dedicated graphics-processing hardware, and so the CPU must perform all graphics operations. The result is that drawing and animating complex, full-color images is slow and difficult to accomplish in real time. Some have solved this problem to a certain extent with the use of specialized assembly-language routines (e.g., Lane \& Ashby, 1987) and, more recently, with the use of off-screen bitmaps to rapidly transfer images to the display screen (Rensink, 1990). However, even with the new off-screen bitmap routines available in Apple's latest system software (see Ortiz, 1990), these real-time display techniques are limited to 1-bit (i.e., black and white) images, relatively small color images, or images composed of relatively few

We would like to thank Apple Computer, Inc., and in particular Ken Pavlicek, a Systems Engineer at Apple's Columbia, MO, office, and the people in the Developer Technical Support group for their assistance in obtaining technical information about the Macintosh. We would also like to thank Rob Dye at National Instruments for his assistance in getting our programs up and running in LabVIEW. Correspondence should be addressed to John A. Baro, School of Optometry, University of Missouri-St. Louis, St. Louis, MO 63121 (e-mail: Bitnet: sjabaro@umslvma). colors. Large, full-color images tend to require too much time to be moved from an off-screen buffer to the display screen. The limiting factor in these approaches is the speed at which the Macintosh's CPU can move the bits that make up the image from one memory location to another. With this type of approach, therefore, the speed at which an image can be displayed or animated is determined by its size and pixel depth (i.e., the number of colors). Even with the relatively fast CPUs available today, large full-color images cannot be moved from an offscreen buffer to display memory at the scan rate of the display, which is typically between 60 and $80 \mathrm{~Hz}$ (e.g., the scan rate of the Apple Color Hi-Resolution RGB monitor is $67 \mathrm{~Hz}$ ).

\section{PALETTE ANIMATION}

The approach presented here relies on the technique of palette animation, a capability built into the Macintosh Toolbox. A similar technique, color cycling, has been reported for the Amiga computer (e.g., see Anstis, 1986), and palette animation on the Macintosh has been discussed in several of Apple's technical publications (e.g., Collyer, 1991; van Brink, 1990). Rather than move the large collection of bits that make up an image from one region of memory to another (each pixel can be represented by 8 or more bits), palette animation changes the colors of images already drawn on the screen. When one or more colors in a palette are changed, creating a new palette, every pixel previously drawn with a particular color in the old palette is quickly (i.e., within one vertical retrace period) changed to its corresponding color in the new palette. Because a palette typically contains substantially fewer entries than there are pixels in an image (palettes are limited to 4,095 colors), images of arbitrary size and pixel depth can be rapidly modified with this technique. 
The discussion of the sample code segments below is limited to the Toolbox routines used in conjunction with palette manipulation and to topics directly relevant to the use of palettes; ${ }^{1}$ basic drawing, timing, and I/O operations and other necessary program components are not included in the examples or discussed in the text. The reader should refer to Inside Macintosh (Apple Computer, $1985,1988 \mathrm{a}, 1991$ ) and the other references for additional information on these topics. It is assumed that the reader has a working knowledge of Macintosh programming, is familiar with the most commonly used Toolbox routines, and knows how to use a Pascal editor/compiler.

\section{System Requirements}

To utilize the palette animation routines presented below, the computer must be equipped with the following: (1) A video card and display monitor capable of displaying at least 256 colors (8-bit video). Palette animation techniques work identically with any video hardware, the only difference being the number of different colors that can be displayed on the screen at the same time. For other applications, 16 colors (4-bit video) may be sufficient. (2) Color QuickDraw. This version of QuickDraw, which is present on all color-capable Macintosh models, provides basic color display capabilities. (3) 32-bit QuickDraw. This is the most recent incarnation of QuickDraw. The routines in 32-bit QuickDraw can utilize up to 24 bits of color information (i.e., $2^{24}$, or $16,777,216$ colors); they also provide capabilities not available in earlier versions of QuickDraw (see Leak, 1990, for a review of the capabilities added to the Toolbox with 32-bit QuickDraw). The three files necessary for 32-bit QuickDraw were released as an upgrade to System Version 6.03 and are installed as an option with versions 6.03 through $6.07 ; 32$-bit QuickDraw is automatically installed with Version 7.0 and later (all sample code segments provided are compatible with System 7.0 and, presumably, later versions). Newer color machines (the IIci and all more recently released models) have 32-bit QuickDraw in ROM, making installation of additional files unnecessary.

The procedure CheckSys presented in Listing 1 can be included in your program to test the machine for these minimum requirements. If any of the requirements are not satisfied, an appropriate error Alert can be presented, as indicated, and the program will terminate with the call ExitToShell. For more information on the Toolbox routines used in this procedure, refer to Macintosh Technical Note No. 129 (Apple Computer, 1987a), Macintosh Technical Note No. 156 (Apple Computer, 1987b), Leak (1990), and Inside Macintosh: Volume V (Apple Computer, 1988a, pp. 5-8).

\section{The Macintosh Color Environment}

Most color display devices utilize a color look-up table (CLUT) to determine what colors are displayed at any given time (refer to Inside Macintosh: Volume VI, chapter 16, for an overview of Macintosh graphics; Apple Computer, 1991). When the system makes a request for a particular color to be displayed (i.e., a particular set of red, green, and blue values to be used in generating a pixel), the device uses the CLUT to find the color that best matches the request and subsequently displays that color. Depending on a number of factors, the match may be exact, close, or a completely different color. If the system requests an exact match or a very close approximation, the CLUT may need to be changed to accommodate the request.

Many of the animation effects described below can be achieved in a similar fashion by using Color Manager routines, which directly manipulate a display device's color environment. Although Color Manager routines can be faster than the higher level Palette Manager routines, using the Color Manager is considerably more complicated and can limit portability, both to other systems with different hardware configurations and to future versions of the operating system. Inside Macintosh recommends that all color manipulations be performed with the Palette Manager, the collection of high-level Toolbox routines devoted to color management. The Palette Manager takes care of all direct, low-level color manipulations, ensuring compatibility and correct color behavior across different machines, systems, and display devices. In addition, the Palette Manager makes the use of multiple monitors transparent. A second monitor is treated as an extension of the first, differing only in the global coordinates used by graphics routines for drawing; no additional or modified code is necessary.

\section{Palettes and the Palette Manager}

When a palette is created, the CLUTs of all active display devices are automatically modified as necessary to best satisfy the requirements of the palette. Because the number of entries in a CLUT is limited by the characteristics of the hardware (e.g., 8-bit video permits a maximum of 256 different colors) the number of different entries in a palette is similarly constrained. However, several palettes can be created and maintained simultaneously, and when a given palette becomes active, all CLUTs are automatically adjusted as necessary to accommodate the new palette.

Palettes can be stored as resources and loaded in by a program as needed, ${ }^{2}$ or they can be created "on the fly" by a program. The example procedures described here demonstrate the programmatic creation of palettes. When a palette is created within a program, its entry values are typically obtained from a structure in memory called a ColorTable (not to be confused with a display device's CLUT). The format of a ColorTable record is described in Inside Macintosh: Volume V (Apple Computer, 1988a, pp. 135-137). The easiest way to create a ColorTable is to use the Toolbox routine GetCTable. GetCTable creates a ColorTable record in memory, loads a CLUT resource into it (see note 2), and returns a handle to the ColorTable. If you wish to modify the colors in the ColorTable record, the values that you must set are in the ctTable field, which contains an array of RGB values 
to be used by the display device (an RGBColor record is made up of three integer values). The creation and modification of a ColorTable is illustrated in the MakeSolidCTable procedure in Listing 2 and in the MakePatternCTable procedure in Listing 3.

By convention, the first entry in a ColorTable, entry 0 , is white (the red, green, and blue fields set to 65535 , the maximum value) and the last entry, 255 in the example, is black (red, green, and blue set to 0 ). These entries should never be changed. This is to ensure compatibility with different versions of QuickDraw, some of which require this color arrangement for drawing objects such as menus and dialog boxes. The other entries in the ColorTable can be set to any combination of red, green, and blue values, $0-65535 .^{3}$ In the example in Listing 2 , all entries are set to the same value; in the example in Listing 3, the values vary in the form of a gray-scale (i.e., the red, green, and blue value of each entry are equal) sine wave.

In the main program (Listing 4), after a color window has been created, the two ColorTables-solidColorTable and patternColorTable-are created with calls to MakeSolidCTable and MakePatternCTable. A palette is then created with a call to NewPalette. The arguments to NewPalette specify the number of entries in the palette, the ColorTable to be loaded into it, a color usage type (in this case, the color usage is set to pmAnimated for animating colors; refer to Collyer, 1991, for additional information about doing palette animation with off-screen pixmaps), and a tolerance level ( 0 indicates that the colors displayed must be an exact match to the colors specified in the palette). The palette is then associated with the current window and activated with SetPalette and ActivatePalette, respectively. At this point, the color environment is changed to reflect the new palette. Colors can now be specified by their relative position, or index, in the palette. For example, white is referred to as entry 0 . Foreground and background colors used in subsequent drawing are specified with the PMForeColor and PMBackColor procedures, which take as their argument an entry number that refers to the current palette. Before the image is drawn, the background color is set and the window is erased. The image can now be drawn with the colors in the current palette. ${ }^{4}$

\section{Animating Palettes}

Notice that the currently active palette at the point in the program where the image is drawn contains the RGB values from solidColorTable, in which all entries (except the first and last) are the same as the background color. As long as colors 0 and 255 are not used in the image, anything drawn with any of the other entries in the palette will be in the same color as the background. In this way, all drawing is done invisibly and therefore will not alter the current display if the drawing time is greater than a single scan period.

Next, to make the invisible image appear, the colors associated with the palette entries used to draw the image must be changed. Two Palette Manager routines can be used to accomplish this. AnimateEntry allows the RGB value of a single palette entry to change, and AnimatePalette, used in the example in Listing 4, allows a number of consecutive palette entries to change at the same time. AnimátePalette takes as its arguments the ColorTable to be loaded into the palette and index values to indicate which palette entries are to be changed. In the example, all palette entries are changed. When AnimatePalette is called, the color of all pixels in the window is immediately (i.e., during the next vertical retrace period ${ }^{5}$ ) changed to reflect the new palette; the image appears. Since the palette entry used to fill in the background, entry 254 , contains the same RGB value in each of the two ColorTables, the background color does not change. If precise timing is important, the call to AnimatePalette should be synchronized with the vertical retrace. Routines for millisecond timing and synchronization with the vertical retrace of the display are provided in Rensink (1990). The image is then made to disappear by reloading the original ColorTable, in which all entries are the background color, into the palette.

The example described above is the simplest case, the use of palette animation to create a tachistoscopic display. However, this technique can be extended to the creation of complex animation sequences. The number of ColorTables that can be created and maintained is limited only by the computer's memory. Several ColorTables can be created and swapped in and out of the palette very rapidly in sequence, creating images in which different areas appear and disappear, or change colors, at different times, creating apparent motion. For example, a periodic pattern drawn with the sine-wave palette created in Listing 3 can be made to drift as follows: Create an array of ColorTables, each of which contains a sine-wave luminance profile that is shifted in phase by $5^{\circ}$ relative to the previous ColorTable in the array. If the program cycles through 72 of these ColorTables, animating the palette with each in turn, the pattern will be shifted by $360^{\circ}$ each time through the cycle. This technique can be used to create a very smooth animated sequence.

If available memory is limited, there are alternatives that utilize memory more efficiently. For example, ColorTables can be created and disposed of as needed. It should also be pointed out that palettes do not always have to contain 256 or more entries; a wide variety of effects can be achieved with 16-color palettes. An alternate, less memory-intensive approach to creating animation sequences involves manipulating the RGB values in a single ColorTable. Since ColorTables typically contain a small number of values relative to the number of pixels in an image, traditional bit manipulation can be done quickly. The procedure in Listing 5 illustrates this type of palette animation technique. The entries in a ColorTable are rotated the number of times specified in the procedure's argument, and the palette is updated after each rotation. The BlockMove procedure (see Inside Macintosh: Volume II, p. 44-Apple Computer, 1985) is called 
to quickly move a block of consecutive bits from one area of memory to another. In this example, the first entry in the ColorTable is stored in a temporary variable, the remaining entries are shifted down one position, and the last entry in the ColorTable is then replaced from the temporary variable. In this way, all palette entries are rotated one position each time through the loop.

\section{CONCLUSIONS}

One area of concern is the speed at which these color animation operations can be performed-that is, whether or not they can be performed in "real time." Because the Macintosh has no dedicated graphics-processing hardware, all graphics operations are performed by the CPU. As a result, palette animation speed is determined by the particular Macintosh model used; models with faster CPUs perform these operations more quickly than models with slower CPUs. The procedures described here have been tested on a number of different CPUs, with an Apple Color Hi-Resolution RGB monitor with 8-bit video, with the following results: ${ }^{6}$ A Macintosh $\Pi x$ or $\Pi c x$ should be considered minimum for acceptable performance. A Macintosh II or LC would probably be too slow for most applications. Our tests indicate that a call to AnimatePalette, with a 256-color palette, takes about $16 \mathrm{msec}$ (i.e., more than a single scan period) to execute on a IIcx. Keep in mind, however, that the device's CLUT is always changed during a single vertical retrace period, so that sync tear (the shearing apart of a moving image that is drawn across two or more scan periods) is never a problem if palette animation is used. At this speed, it is therefore possible to maintain a coherent, moving image at a frame rate of up to $30 \mathrm{~Hz}$. We have also tested these routines on a Ici, a Isi, and a Ifx. Each of these machines can execute the same call to AnimatePalette in less than $15 \mathrm{msec}$ (the scan rate of our monitor). Of course, faster machines execute the call faster, leaving more time for other operations within the animation loop. On a IIsi, there is little time (about $2 \mathrm{msec}$ ) for anything else if one wishes to change the display at $60 \mathrm{~Hz}$; on a Iffx, only a small portion of each 15-msec scan period is occupied by the call to AnimatePalette.

Given these constraints, the techniques described in this paper can be used to create sophisticated, full-color animation sequences in real time on the Macintosh display. The complexity of the animation is limited only by the programmer's creativity in designing palettes. The example program and procedures can serve as a palette animation skeleton; the remaining graphics, timing, and I/O portions of the program can be filled in to complete the program.

\section{REFERENCES}

ANSTis, S. (1986). Visual stimuli on the Commodore Amiga: A tutorial. Behavior Research Methods, Instruments, \& Computers, 18, 535-541. APPLE COMPUTER (1985). Inside Macintosh: Volumes I-III. Menlo Park, CA: Addison-Wesley.
APple COMPUter (1987a). Macintosh technical note No. I29. Cupertino, CA: Author. (Rev. 1990)

APPLE CoMpUTER (1987b). Macintosh technical note No. 156. Cupertino, CA: Author. (Rev. 1988)

APPLE Computer (1988a). Inside Macintosh: Volume V. Menlo Park, CA: Addison-Wesley.

APPLE Computer (1988b). Macintosh technical note No. 211. Cupertino, CA: Author.

APPLE CoMPUTER (1991). Inside Macintosh: Volume VI. Menlo Park, CA: Addison-Wesley.

Collyer, R. (1991). Palette manager animation. Develop, 5 (February), 78-84.

LANE, D. M., Ashry, B. (1987). PsychLib: A library of machine language routines for controlling psychology experiments on the Apple Macintosh computer. Behavior Research Methods, Instruments, \& Computers, 19, 246-248.

LEAK, B. (1990). Realistic color for real-world applications. Develop, 1 (January), 4-21.

OrTIZ, G. (1990). Braving offscreen worlds. Develop, 1 (January), 28-40.

Rensinx, R. A. (1990). Toolbox-based routines for Macintosh timing and display. Behavior Research Methods, Instruments, \& Computers, 22, $105-117$

SHEETS, S. (1988). Color screen dump FKEY. MacTutor, 4 (2), 52-60. VAN Brink, D. (1990). All about the palette manager. Develop, 1 (January), 22-27

\section{NOTES}

1. The source code presented here was written in Think Pascal, Version 3.02 (Symantec Corp., Cupertino, CA). Additional information about the Palette Manager routines used in the examples can be found in Inside Macintosh: Volume V, chapter 7 (Apple Computer, 1988), Inside Macintosh: Volume $V$, chapter 20 (Apple Computer, 1991), Macintosh Technical Note No. 211 (Apple Computer, 1988b), Collyer (1991), and van Brink (1990).

2. Resources are objects used by programs and are stored with programs, but they are "separate" in that they can be modified without recompiling the program. Almost everything that is stored in a Macintosh is, or can be, a resource. Standard Macintosh resource types include windows, dialog boxes, cursors, icons, menus, and even the program code itself.

Palette, ColorTable, and other resources can be created with a resource editor (e.g., ResEdit, Version 2.1, Apple Computer, Inc.). The formats of these resources are described in Inside Macintosh: Volume $V$, pages 81 and 160-161 (Apple Computer, 1988). In addition, there are a number of system default CLUT resources available at all times. The default ColorTables have resource numbers that correspond to their pixel depth (e.g., an 8-bit ColorTable is specified with a CLUT resource ID of 8). Default gray-scale ColorTables can be specified by adding 32 to the pixel depth (e.g., an 8-bit gray ramp is specified with a resource ID of 40). Refer to van Brink (1990) and Inside Macintosh: Volume VI (Apple Computer, 1991) for more details.

3. If the Think Pascal compiler is used, setting values in a ColorTable record in this way (i.e., a dynamically created data structure referenced with a handle) will generate a range error during compilation. This is because the compiler has no way of knowing how many elements have been allocated for the array. To avoid this problem, use the \$PUSH and \$R-compiler directives (as shown in Listings 3 and 4) to save current compiler options and turn off range checking, respectively, and then the \$POP directive to restore the saved compiler options.

4. If the image is too complex to be drawn quickly or if one wishes to import a picture created by another application (i.e., a file saved in the PICT2 format), one will probably want to use the palette with which the picture was originally created. A routine that can be used to extract a ColorTable from a PICT file can be found in Sheets (1988), and a routine that can be used to spool in a PICT file from disk is described in Inside Macintosh: Volume V (Apple Computer, 1988, pp. 87-89).

5. The AnimatePalette procedure performs a number of palettemanagement functions in addition to actually changing the CLUTs of 
current devices. The last thing AnimatePalette does is call SetEntries, a Color Manager routine, which is the call responsible for changing the entries in a device's CLUT. To avoid color artifacts from changing colors while the screen is being redrawn, SetEntries always waits for the next vertical blanking interrupt before proceeding (personal communication, Apple Developer Technical Support).

6. The timing information discussed here should be taken as an approximation, at best. A number of factors, both in hardware and soft- ware, can affect the time that it takes to execute any given procedure. One factor that has a significant effect on the speed of palette animation is the number of monitors. The addition of a second monitor will double the execution times reported here. It should also be pointed out that a QuickDraw accelerator will not affect the speed at which Palette Manager routines are executed; the Palette Manager and Color Manager are not part of QuickDraw and so are not accelerated (personal communication, Apple Developer Technical Support).

\section{LISTING 1}

The CheckSys procedure is used to determine whether the machine has the hardware and system software necessary for using the Palette Manager routines.

procedure CheckSys; [Check System environment and display characteristics]

const

QD32Trap = \$AB03;

UnImplTrap $=\$$ A89F; var

ColorQDExists, QD32Exists: boolean;

MainDevice: GDHandle; $\quad$ [Handle to graphics device returned as main monitor]

myEnvRec: SysEnvRec; $\quad$ [A system environment record]
[Trap address for 32-bit QuickDraw routines \}

[Trap address returned by NGetTrapAddress if trap does not exist]

$$
\text { err: OSErr; }
$$

\section{begin}

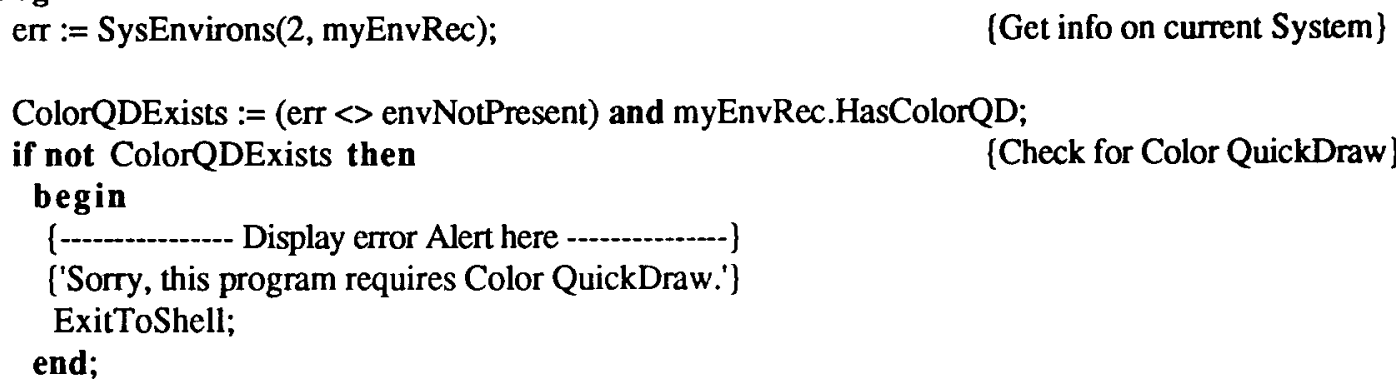

end; [CheckSys\} 
LISTING 2

The MakeSolidCTable procedure creates a ColorTable structure in memory and loads the default 8-bit grayscale ColorTable into it. By convention, the first entry in the ColorTable is white and the last entry is black. In this example, the ColorTable is modified so that the remainder is filled with the same color, a medium gray. The procedure SetGrayColor is called to set the red, green, and blue fields of an RGBColor record to the same value, creating a shade of gray.

function SetGrayColor (grayColor: longint): RGBColor; (Assigns the same value to the red, green, \& blue \}

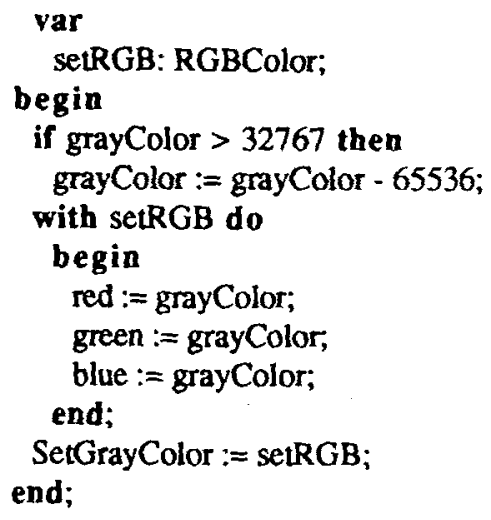

(An RGB color record)

[We need to represent values from 0 to 65535 with)

$\{$ integers. Pascal integers range from -32768 to 32767 .

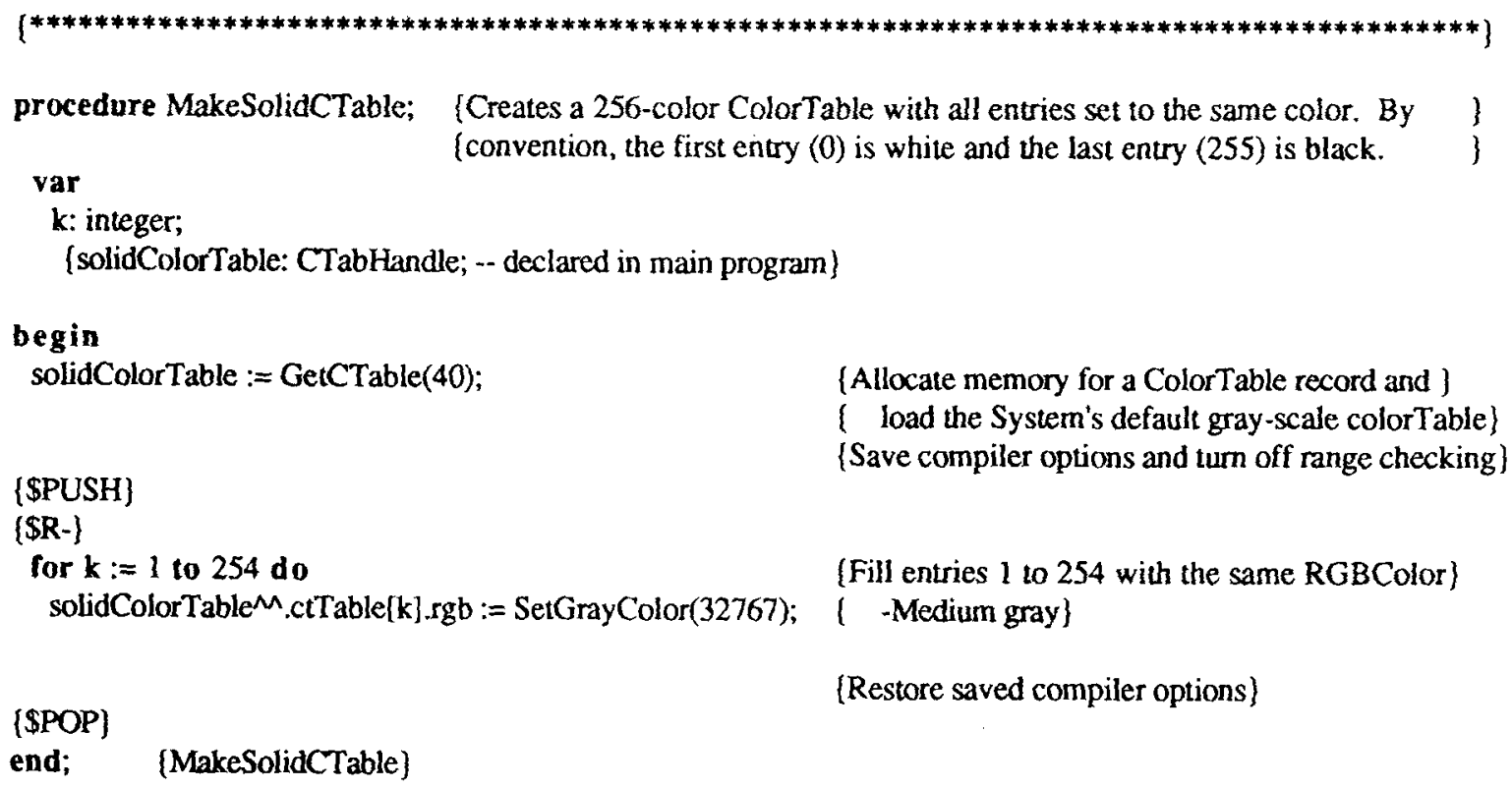

[Restore saved compiler options\}

(Allocate memory for a ColorTable record and \} ( load the System's default gray-scale colorTable [Save compiler options and turn off range checking] 


\section{LISTING 3}

The MakePatternCTable procedure is similar to the procedure shown in Listing 2, with the exception that the ColorTable is filled with a range of gray-scale colors. In this example, the shades of gray vary sinusoidally, creating gradual transitions from dark to light (a periodic pattern known as a sine wave).

procedure MakePattemCTable; (Creates a 256-color ColorTable. By convention the first entry $(0)$ is white and (the last entry (255) is black. Entries 1-253 are set to a sine-wave gray-scale (map and entry 254 is set to the background color.

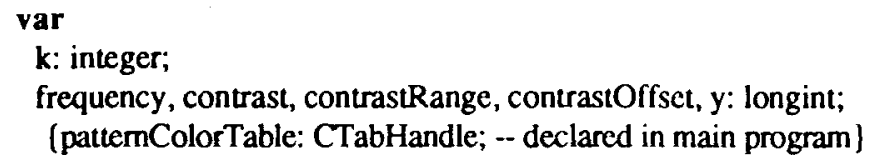

\section{LISTING 4}

This is the main program segment that calls the procedures shown in Listings 1, 2, and 3. First, CheckSys is called to check the color environment; then, a color window that is slightly larger than the screen is created. Next, the two ColorTable creation procedures are called, and a palette is created, filled with the solid-color ColorTable, and activated. The image is then made to appear by animating the palette with the patterned ColorTable, and it then disappears when the palette is again animated with the solid-color ColorTable. Finally, the handles that point to the palette and the two ColorTables are disposed of, freeing up memory for other uses, and the System's default ColorTable is restored. (Note: If the Think Pascal compiler is used, the Palettes.p interface must be included in the project and in the program's "uses"clause in order to call Palette Manager routines.)

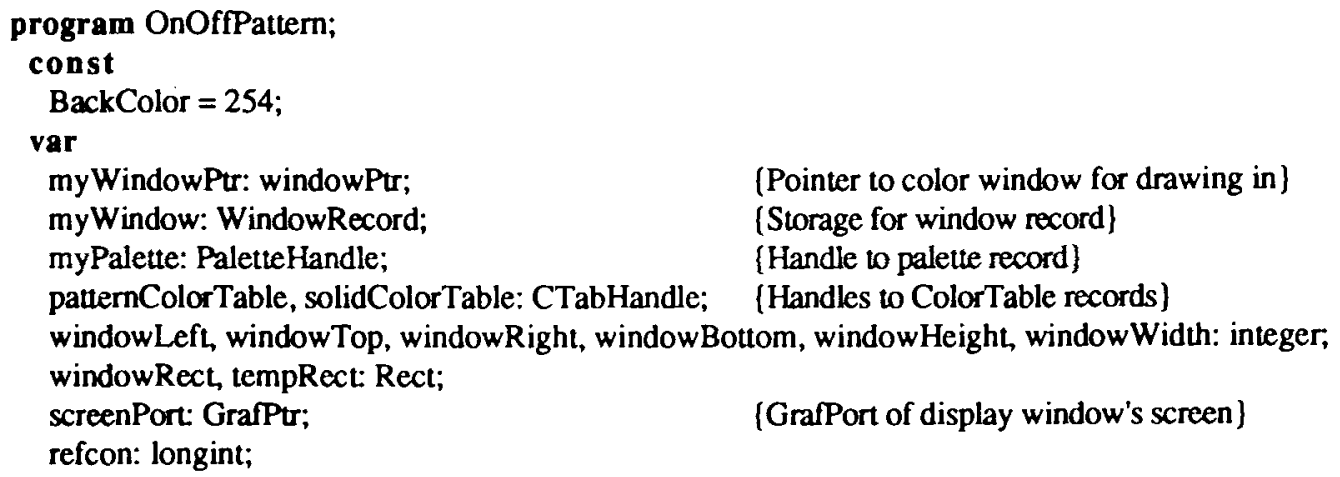

(Pointer to color window for drawing in)

[Storage for window record]

(Handle to palette record)

[Handles to ColorTable records)

[GrafPort of display window's screen ] 


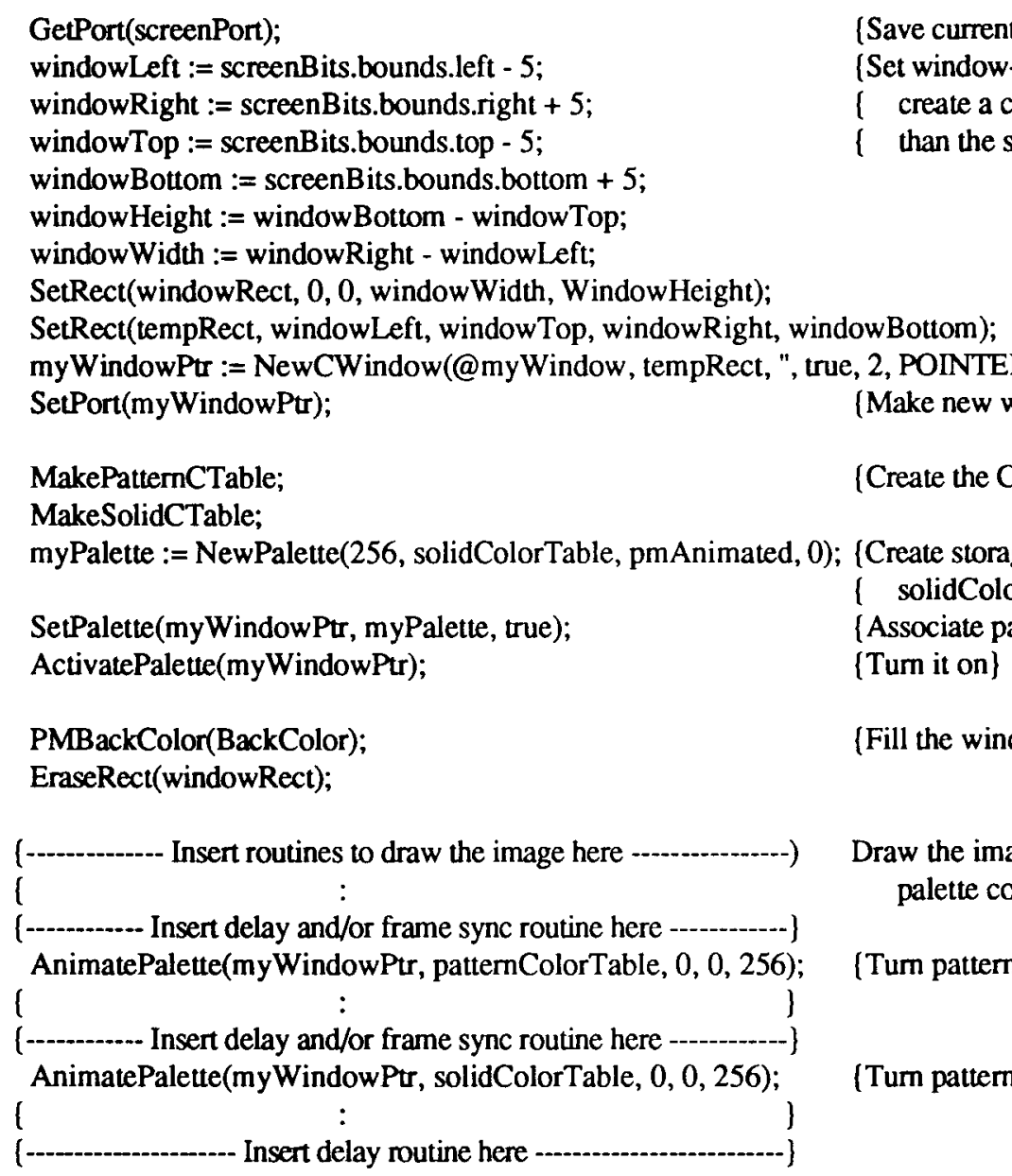

\{Fill the window with the background color\}

Draw the image while all entries in the current palette contain the same color

[Turn pattern on - swap ColorTables]

[Tum pattern off - swap ColorTables]
DisposePalette(myPalette);

DisposCTable(solidColorTable);

DisposCTable(pattemColorTable);

RestoreDeviceCLUT(nil);

SetPort(screenPort);

end. [OnOffPattern]
[Free memory allocated for palette and)

\{ ColorTable records\}

\{Restore System's ColorTable so other programs display properly] [Restore saved GrafPort\} 


\section{LISTING 5}

The RotateCTable procedure rotates the entries in a ColorTable the number of times specified in the argument passed to it. After each rotation, the current palette is animated with the modified ColorTable, making the changes appear on screen.

procedure RotateCTable (repeatTimes: integer); (Rotate entries 1-253 of palette, leave entries 0, 254-255 intact. )

(Put the new ColorTable into current palette after each rotation. ]

var

k: integer;

tempCSpec: ColorSpec;

(pattemColorTable: CTabHandle; -- declared in main program)

[an entry in a ColorTable)

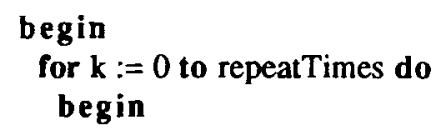

[\$PUSH]

[Save compiler options and tum off range checking]

[\$R-]

tempCSpec := patternColorTable ${ }^{\wedge}$. ctTable $[1] ;$

[Save entry 1 in temporary variable]

[Shift entries 2-253 down 1 position]

BlockMove(@patternColorTable ${ }^{M} . c t T a b l e[2], @ p a t t e m C o l o r T a b l{ }^{M} . c t T a b l e[1], 252 *$ SizeOf(ColorSpec));

pattemColorTable^^.ctTable[253] := tempCSpec;

[Replace entry 253 with temporary variable\}

[ i.e., previous contents of entry 1 \}

\{Restore saved compiler options\}

[\$POP\}

[---- Insert delay and/or frame sync routine here -........-.-]

AnimatePalette(myWindowPtr, patternColorTable, 0, 0, 256); [Put new, rotated ColorTable into current palette) end;

end; \{RotateCTable $\}$

(Manuscript received February 25, 1991;

revision accepted for publication July 10, 1991.)

\section{Erratum}

Gillett, R. A FORTRAN 77 program for sample-size determination in replication attempts when effect size is uncertain. Behavior Research Methods, Instruments, \& Computers, 1991, 23, 442446-The program is able to provide sample sizes for both one-sample and two-sample tests. As written, it covers the two-sample case. To obtain the sample size for a one-sample test, simply change 2.0 to 1.0 in the line

$$
\text { IF (V.LT.99) } \mathrm{W}=\mathrm{V} * \mathrm{AN} /(\mathrm{V} * \mathrm{AN}+2.0)
$$

in the subroutine SAMPSIZE on page 445 , so that it now reads

$$
\text { IF (V.LT.99) } \mathrm{W}=\mathrm{V}^{*} \mathrm{AN} /(\mathrm{V} * \mathrm{AN}+1.0) \text {. }
$$

